\title{
Instrumento para medir variables psicológicas y comportamientos de adhesión al tratamiento en personas seropositivas frente al VIH (VPAD-24)
}

\author{
Julio Alfonso Piña López, ${ }^{1}$ Andrés Ernesto Corrales Rascón, ${ }^{2}$ \\ Katherine Mungaray Padilla ${ }^{3}$ y Manuel Alejandro Valencia Vidrio ${ }^{3}$
}

Forma de citar

Piña López JA, Corrales Rascón AE, Mungaray Padilla K, Valencia Vidrio MA. Instrumento para medir variables psicológicas y comportamientos de adhesión al tratamiento en personas seropositivas frente al VIH (VPAD-24). Rev Panam Salud Publica. 2006;19(4):217-28.

RESUMEN Objetivo. Probar las propiedades psicométricas de un instrumento que mide variables psicológicas y comportamientos de adhesión al tratamiento en personas con VIH/sida.

Métodos. Se realizó un estudio transversal que contó con la participación de 68 personas seropositivas frente al virus de la inmunodeficiencia humana (VIH) que en el momento del estudio recibían atención en una institución de salud de la ciudad de Hermosillo, México. Para el análisis de los datos se utilizaron cinco procedimientos estadísticos en orden sucesivo: la prueba de la $\mathrm{t}$ de Student de comparación de medias, para evaluar la comprensión de las preguntas; un análisis factorial exploratorio con rotación varimax, para estudiar la validez conceptual o teórica del instrumento (construct validity); el coeficiente $\alpha$ de Cronbach, para valorar la confiabilidad; el coeficiente de correlación de Pearson (r), para analizar la validez concurrente, y un análisis de regresión lineal múltiple, para estimar la validez predictiva.

Resultados. Con la prueba de la $\mathrm{t}$ de Student de comparación de medias se obtuvieron valores de significación estadística de $\mathrm{P} \leq 0,05, \mathrm{P} \leq 0,005, \mathrm{P}<0,01$ y $\mathrm{P}<0,001$ en 25 de las 30 preguntas. El análisis factorial exploratorio con rotación varimax arrojó una estructura de cuatro factores: 1) comportamientos de adhesión presentes y tratamiento; 2) motivos que subyacen a los comportamientos de adhesión o falta de adhesión; 3) bienestar psicológico y competencias sociales y 4) comportamientos de adhesión pasados con valores propios superiores a 1 y que en conjunto explican 65,50\% de la varianza. En el análisis de confiabilidad se obtuvo un coeficiente $\alpha$ global de 0,852 . En el estudio de la validez concurrente se hallaron correlaciones entre los factores 1 y $2(\mathrm{r}=0,615 ; \mathrm{P}<0,01)$, entre el factor 2 y los factores 3 y 4 $(\mathrm{r}=-0,178 ; \mathrm{P}<0,05$ y $\mathrm{r}=0,265 ; \mathrm{P}<0,05$, respectivamente), así como entre el factor $3 \mathrm{y}$ la variable tiempo de infección en meses $(\mathrm{r}=0,265 ; \mathrm{P}<0,05)$. Por último, el análisis de regresión lineal múltiple mostró que los factores psicológicos 2 y 4 -que en conjunto explican el 48,6\% de la varianza- predijeron los comportamientos de adhesión en el presente (coeficiente de determinación $\left[\mathrm{R}^{2}\right]$ ajustado $\left.=0,486\right)$.

Conclusión. Según los resultados obtenidos, el instrumento posee una confiabilidad óptima y validez en sus tres variantes: conceptual, concurrente y predictiva.

Palabras clave Cooperación del paciente, seropositividad, confiabilidad de resultados, validez, México.

Consultor privado, Hermosillo, Sonora, México. Toda correspondencia deberá dirigirse a: Julio Alfonso Piña López, Guillermo Prieto 18, Col. Cons- titución. Hermosillo, Sonora, C. P. 83150, México. Correo electrónico: jpina@salud.gob.mx

2 Centro de Información para la Salud, Secretaría de Salud Pública del estado de Sonora, México.
3 Escuela de Psicología, Universidad de Sonora, Hermosillo, México. 
En diferentes investigaciones (1-4) se ha demostrado que el tratamiento con medicamentos antirretrovirales reduce significativamente las tasas de morbilidad y mortalidad asociadas con la infección por el VIH y el síndrome de inmunodeficiencia adquirida (sida). Sin embargo, considerando que dicho tratamiento suele resultar bastante complejo para las personas infectadas por el VIH, es imprescindible que tanto el diseño como la conducción del plan de intervención se lleven al cabo en instituciones con experiencia, bajo la dirección de un equipo interdisciplinario (5), dentro del cual el profesional de la psicología ocupa un lugar especial (6-9).

Así, hoy en día se sabe, por ejemplo, que los pacientes que enfrentan problemas emocionales - en especial, del tipo de los que se describen tradicionalmente en términos de estrés, ansiedad, depresión, miedo e impulsividad (10-13) - y no cuentan con redes de apoyo social (14-16), o aquellos que presentan déficit cognoscitivos (17) son los que menos probabilidades tienen de cumplir el tratamiento en los porcentajes esperados, superiores al umbral del $90 \%$ considerado como el mínimo que deben alcanzar las personas infectadas por el VIH para evitar el avance clínico de la enfermedad (18-20) Contrariamente a lo que pudiera pensarse, esto constituye un serio problema para el equipo interdisciplinario, pues exige el diseño y la instrumentación de estrategias de intervención eficaces, cuyo objetivo final es favorecer la práctica de comportamientos de adhesión al tratamiento (21-23).

Otro problema que en la actualidad está llamando la atención de las autoridades sanitarias de todo el mundo y la de los propios profesionales de la salud (24-26) es la medición de los comportamientos de adhesión. En efecto, en contraposición con lo que ocurre en los países desarrollados, en los cuales para evaluar estos comportamientos se recurre incluso a recursos tecnológicos altamente especializados - como el llamado sistema de monitoreo electrónico (MEMS, por sus siglas en inglés) (27)—, en los países como el nuestro ha sido generalmente notoria la carencia de investigaciones realizadas para tal efecto (28-29).

De esto deriva justamente la importancia de que como profesionales de la psicología interesados en el campo de los problemas de la salud participemos en investigaciones de corte interdisciplinario orientadas hacia la evaluación de los comportamientos de adhesión en personas infectadas por el $\mathrm{VIH}$. Las razones de hacerlo tienen que ver con la imperiosa necesidad, una vez obtenidos determinados resultados, de estar en una mejor posición para coadyuvar en el diseño y la instrumentación de programas de intervención en los términos expuestos previamente. Estamos obligados, en consecuencia, no solo a disminuir el sufrimiento de quienes están infectados por el VIH, sino también a procurar mejorar su calidad de vida durante el mayor tiempo posible.

Partiendo de lo expuesto y aplicando un modelo psicológico de salud biológica (30-31), se planteó el presente trabajo con el objetivo de probar las propiedades psicométricas (confiabilidad y validez - conceptual, concurrente y predictiva-) de un instrumento que mide variables psicológicas y comportamientos de adhesión al tratamiento en personas seropositivas.

\section{MATERIALES Y MÉTODOS}

Previa autorización del Comité de Investigación de la Academia de Psicología Clínica y Salud de la Universidad de Sonora y del Comité de Investigación y Ética de la Secretaría de Salud Pública del estado de Sonora, se realizó en el transcurso del mes de noviembre de 2005 el presente estudio de corte transversal, en el que participaron 68 personas seropositivas. Ello correspondió a poco más del $75 \%$ del total de pacientes que en el momento del estudio recibían atención en una institución sanitaria dependiente de la Secretaría de Salud Pública del estado de Sonora y se encontraban en tratamiento con medicamentos antirretrovirales. La edad media de los participantes era de 36,4 años (desviación estándar [DE] =
10,64), 46 eran de sexo masculino y 22, de sexo femenino (véase el cuadro 1 , donde se describen las variables sociodemográficas y clínicas del conjunto de los participantes).

Antes de comenzar a utilizar el instrumento, se solicitó el consentimiento válido de cada uno de los participantes, en un documento donde se describían las características y los objetivos del estudio. Además, se aclaró a todos los pacientes que sus respuestas serían anónimas y confidenciales y que los resultados del estudio se utilizarían para coadyuvar en la elaboración y la instrumentación de programas de intervención interdisciplinaria orientados a:

a) favorecer la práctica de comportamientos de adhesión al tratamiento;

b) mejorar su desempeño competencial y motivacional frente a los requerimientos impuestos por la enfermedad, $y$

c) promover la práctica de comportamientos saludables de diferentes tipos y mejorar su calidad de vida.

El instrumento utilizado (anexo 1) fue elaborado por el primer autor del presente artículo, a partir de los supuestos de un modelo psicológico de salud biológica (30); en su versión original constaba de 30 preguntas, las cuales se agruparon en tres apartados.

El primer apartado contenía cinco preguntas sobre la práctica de comportamientos de adhesión al tratamiento anteriores al diagnóstico de la infección por el VIH. Por ejemplo: "Antes de que usted recibiera el diagnóstico como VIH positiva, si por algún motivo se sentía mal o enfermaba físicamente, ¿acudía con un médico para que lo revisara y le diera tratamiento?", con opciones de respuesta en un formato de Likert que iban de 1 (nunca) a 5 (siempre).

El segundo apartado contenía 13 preguntas relacionadas con la práctica de comportamientos de adhesión al tratamiento posteriores al diagnóstico de la infección por el VIH y con los motivos que subyacen tras estos comportamientos. Por ejemplo: "En el transcurso de la semana pasada, ¿consumió 
CUADRO 1. Características sociodemográficas y clínicas de los participantes

\begin{tabular}{|c|c|c|c|c|c|}
\hline & $n$ & $\%$ & Media & $\mathrm{DE}^{\mathrm{a}}$ & Mín./Máx. \\
\hline \multicolumn{6}{|l|}{ Sexo: } \\
\hline Hombres & 46 & 67,6 & & & \\
\hline Mujeres & 22 & 32,4 & & & \\
\hline Edad (años): & & & 36,4 & 10,64 & $20 / 69$ \\
\hline \multicolumn{6}{|l|}{ Estado civil: } \\
\hline Soltera/o & 37 & 54,4 & & & \\
\hline Casada/o & 9 & 13,2 & & & \\
\hline Unión libre & 15 & 22,4 & & & \\
\hline Separada/o & 5 & 7,3 & & & \\
\hline Viuda/o & 2 & 2,9 & & & \\
\hline \multicolumn{6}{|l|}{ Nivel de estudios: } \\
\hline Ninguno & 2 & 2,9 & & & \\
\hline Primario & 24 & 35,9 & & & \\
\hline Secundario & 23 & 33,8 & & & \\
\hline Bachillerato & 11 & 14,7 & & & \\
\hline Universitario & 8 & 11,7 & & & \\
\hline \multicolumn{6}{|l|}{ Tiempo de infección: } \\
\hline (en meses) & & & 49,9 & 39,1 & $6 / 157$ \\
\hline \multicolumn{6}{|l|}{ Carga viral: } \\
\hline$<400$ copias $/ \mathrm{mL}$ & 22 & 32,35 & & & \\
\hline 401-30 000 copias $/ \mathrm{mL}$ & 30 & 44,11 & & & \\
\hline$>30000$ copias $/ \mathrm{mL}$ & 16 & 23,53 & & & \\
\hline \multicolumn{6}{|l|}{ Linfocitos T CD4+: } \\
\hline$<200$ células/mL & 45 & 66,17 & & & \\
\hline 201-400 células/mL & 13 & 26,47 & & & \\
\hline >400 células $/ \mathrm{mL}$ & 5 & 7,36 & & & \\
\hline
\end{tabular}

${ }^{a} \mathrm{DE}$ : desviación estándar.

usted los medicamentos tal y como se lo indicó su médico?", con opciones de respuesta en un formato de Likert que iban de 1 (ningún día de la semana) a 5 (todos los días de la semana); a continuación, se preguntaba: "Si usted consumió todos los medicamentos los siete días de la semana pasada, ¿nos podría decir el motivo de por qué lo hizo y qué tan determinante fue cada uno?", con motivos que contemplaban "He experimentado sus beneficios" o "Lo hago porque deseo mantenerme saludable el mayor tiempo posible", con opciones de respuesta en un formato de Likert que iban de 1 (no fue un motivo determinante) a 4 (fue un motivo determinante).

El tercer apartado contenía cuatro preguntas sobre la evaluación de las competencias de los participantes respecto de diversos asuntos relacionados con la infección por el VIH y el tratamiento. Por ejemplo: “Cómo evalúa la información que tiene sobre los medicamentos que consume actualmente?", con opciones de respuesta en un formato de Likert que iban de 1 (no tengo información) a 5 (bastante completa).

Por último, el cuarto apartado contenía ocho preguntas sobre el grado de dificultad que los participantes percibían para practicar diferentes comportamientos. Por ejemplo: “¿Qué tan difícil se le hace a usted buscar información sobre el problema del VIH/ SIDA, el tratamiento, los estudios de laboratorio, etcétera?", con opciones de respuesta en un formato de Likert que iban de 1 (bastante difícil) a 5 (nada difícil).

Para probar las propiedades psicométricas del instrumento, los datos se cargaron en el paquete estadístico SPSS para Windows, versión 10.0, con el cual se extrajeron las frecuencias y los porcentajes de respuesta a cada una de las preguntas. Con el objeto de probar la dificultad de cada una de estas, las preguntas se agruparon según las opciones de respuesta que incluían (es decir, cuatro, en el caso de los motivos, o cinco, para el resto de las preguntas), utilizando para ello la prueba de la $t$ de Student para comparar dos situaciones o muestras, independientes o relacionadas. Tras agrupar las preguntas - por ejemplo, las del apartado de comportamientos de adhesión en el pasado-, se sumaron para cada una de ellas las puntuaciones totales obtenidas por el conjunto de participantes, extrayéndose de un análisis de frecuencia los valores más bajos -inferiores o iguales al primer cuartil (percentil 25) — y más altos superiores o iguales al tercer cuartil (percentil 75)—. Se formaron así dos grupos (valores bajos y altos), que se analizaron respecto de cada una de las preguntas con la citada prueba.

A continuación se procedió a probar la validez conceptual o teórica del instrumento (construct validity), para lo cual se llevó a cabo un análisis factorial exploratorio (método de componentes principales) con rotación varimax (normalización de Kaiser), partiendo de un criterio triple: únicamente se tomaron en cuenta las preguntas que 1) habían originado diferencias estadísticamente significativas en el análisis previo, 2) alcanzaron pesos factoriales iguales o superiores a 0,40 y 3) figuraron en un solo factor. Este procedimiento de extracción permite identificar la manera en que las variables de interés se agrupan en uno o más factores, que se presume comparten determinadas premisas teóricas y conceptuales.

Posteriormente, se realizó un análisis de confiabilidad, para lo cual se calculó el coeficiente $\alpha$ de Cronbach, considerando las variables en su conjunto $\mathrm{y}$, adicionalmente, tal y como quedaron agrupadas en cada uno de los factores. Por otro lado, se demostró la validez concurrente del instrumento calculando el coeficiente de correlación de Pearson $(r)$, con el que se pretende establecer si las medidas de interés cambian cuando lo hacen medidas relacionadas entre variables específicas o entre variables agrupadas en factores; para los propósitos de este trabajo, se tuvieron en cuenta las correlaciones entre las variables agrupadas en los diferentes factores. Por último, la validez predictiva se probó 
mediante un análisis de regresión lineal múltiple, que permite identificar si una o más variables específicas o agrupadas en factores predicen o "causan" otras variables (32-34).

\section{RESULTADOS}

Tal y como se observa en el cuadro 2 , únicamente cinco de las 30 preguntas presentaron dificultades de comprensión, esto es, no originaron diferencias significativas: la número 15 , "no consumo de medicamentos por malestar físico o psicológico" $(t=$ -0,139; $P=0,890$ ); la 19, "evaluación de la información sobre la enfermedad" ( $t=0,538 ; P=0,529) ;$ la 20 , "evaluación de la información sobre el tratamiento" ( $t=-1,355 ; P=0,188)$; la 21, "evaluación de la información sobre los estudios y los resultados de laboratorio" ( $t=-1,255 ; P=0,216) ; y$ la 22, "evaluación de la información que proporciona el médico" $(t=$ $-1,721 ; P=0,690$ ).

En el cuadro 3 se resumen los resultados del análisis factorial exploratorio. Se formaron cuatro factores, cada uno con valores propios superiores a 1 , que en conjunto explican $65,50 \%$ de la varianza total. La medida de adecuación de la muestra de Kaiser-Meyer-Olkin fue de 0,612 , mientras que la prueba de esfericidad de Bartlett dio un resultado significativo $\left(\chi^{2}=1602,021\right.$, grados de libertad $=300, P=0,000$ ). Por lo que hace a la confiabilidad, el $\alpha$ de Cronbach global del instrumento fue de 0,852; cabe mencionar que con este procedimiento se eliminó una pregunta más, la número 23, "búsqueda de información sobre la enfermedad y el tratamiento", por lo que la versión definitiva del instrumento contiene 24 preguntas (VPAD-24).

En el cuadro 4 se describen los resultados del análisis de la validez concurrente. En términos generales, se observaron correlaciones entre los factores relativos a variables psicológicas, y entre uno de ellos, el factor 3 (bienestar psicológico y competencias sociales) y una de las variables biológicas (el tiempo de infección en meses): el factor 1 se correlacionó con el factor 2
CUADRO 2. Análisis de la dificultad de los ítems mediante la prueba de la $t$ de Student

\begin{tabular}{|c|c|c|c|c|c|c|c|}
\hline Ítems & Grupos & $n$ & Media & $\mathrm{DE}^{\mathrm{a}}$ & $t$ & $G L^{b}$ & $P$ \\
\hline \multirow[t]{2}{*}{1} & 1 & 14 & 1,86 & 1,23 & $-6,215$ & 31 & 0,000 \\
\hline & 2 & 19 & 4,16 & 0,90 & & & \\
\hline \multirow[t]{2}{*}{2} & 1 & 14 & 2,00 & 1,36 & $-9,011$ & 31 & 0,000 \\
\hline & 2 & 19 & 4,89 & 0,32 & & & \\
\hline \multirow[t]{2}{*}{3} & 1 & 14 & 1,58 & 0,85 & $-10,634$ & 31 & 0,000 \\
\hline & 2 & 19 & 4,53 & 0,77 & & & \\
\hline \multirow[t]{2}{*}{4} & 1 & 14 & 2,07 & 1,14 & $-7,944$ & 31 & 0,000 \\
\hline & 2 & 19 & 4,68 & 0,71 & & & \\
\hline \multirow[t]{2}{*}{5} & 1 & 14 & 2,50 & 1,56 & $-6,561$ & 31 & 0,000 \\
\hline & 2 & 19 & 4,89 & 0,32 & & & \\
\hline \multirow[t]{2}{*}{6} & 1 & 14 & 3,79 & 1,53 & $-3,001$ & 31 & 0,005 \\
\hline & 2 & 19 & 4,89 & 0,46 & & & \\
\hline \multirow[t]{2}{*}{7} & 1 & 51 & 0,96 & 0,20 & $-1,950$ & 66 & 0,052 \\
\hline & 2 & 17 & 0,82 & 0,88 & & & \\
\hline \multirow[t]{2}{*}{8} & 1 & 51 & 0,96 & 0,20 & $-2,124$ & 66 & 0,031 \\
\hline & 2 & 17 & 1,29 & 1,31 & & & \\
\hline \multirow[t]{2}{*}{9} & 1 & 51 & 0,98 & 0,24 & $-2,954$ & 66 & 0,004 \\
\hline & 2 & 17 & 1,65 & 1,58 & & & \\
\hline \multirow[t]{2}{*}{10} & 1 & 51 & 1,08 & 0,39 & $-2,297$ & 66 & 0,025 \\
\hline & 2 & 17 & 1,65 & 1,66 & & & \\
\hline \multirow[t]{2}{*}{11} & 1 & 51 & 3,92 & 0,20 & $-5,983$ & 66 & 0,000 \\
\hline & 2 & 17 & 1,47 & 1,70 & & & \\
\hline \multirow[t]{2}{*}{12} & 1 & 51 & 5,88 & 0,31 & $-5,045$ & 66 & 0,000 \\
\hline & 2 & 17 & 1,18 & 1,55 & & & \\
\hline \multirow[t]{2}{*}{13} & 1 & 51 & 3,92 & 0,20 & $-5,742$ & 66 & 0,000 \\
\hline & 2 & 17 & 1,12 & 1,32 & & & \\
\hline \multirow[t]{2}{*}{14} & 1 & 51 & 3,92 & 0,20 & $-5,642$ & 66 & 0,000 \\
\hline & 2 & 17 & 1,47 & 1,81 & & & \\
\hline \multirow[t]{2}{*}{15} & 1 & 14 & 4,43 & 1,16 & $-0,139$ & 31 & 0,890 \\
\hline & 2 & 19 & 4,47 & 0,70 & & & \\
\hline 16 & 1 & 14 & 4,17 & 1,09 & $-1,991$ & 31 & 0,006 \\
\hline & 2 & 19 & 4,57 & 0,84 & & & \\
\hline 17 & 1 & 14 & 4,07 & 1,41 & $-1,865$ & 31 & 0,048 \\
\hline & 2 & 19 & 4,68 & 0,58 & & & \\
\hline 18 & 1 & 14 & 4,36 & 1,01 & $-1,842$ & 31 & 0,053 \\
\hline & 2 & 19 & 4,79 & 0,42 & & & \\
\hline 19 & 1 & 14 & 4,07 & 1,07 & 0,538 & 66 & 0,592 \\
\hline & 2 & 54 & 4,26 & 1,18 & & & \\
\hline 20 & 1 & 14 & 4,21 & 1,25 & $-1,355$ & 66 & 0,180 \\
\hline & 2 & 54 & 4,63 & 0,90 & & & \\
\hline 21 & 1 & 14 & 4,14 & 1,23 & $-1,255$ & 66 & 0,216 \\
\hline & 2 & 54 & 4,54 & 1,00 & & & \\
\hline 22 & 1 & 14 & 4,00 & 1,36 & $-1,721$ & 66 & 0,090 \\
\hline & 2 & 54 & 4,54 & 0,95 & & & \\
\hline 23 & 1 & 16 & 3,31 & 1,25 & $-5,494$ & 37 & 0,000 \\
\hline & 2 & 23 & 4,87 & 0,46 & & & \\
\hline 24 & 1 & 16 & 3,69 & 1,62 & $-3,095$ & 37 & 0,000 \\
\hline & 2 & 23 & 5,00 & 0,00 & & & \\
\hline 25 & 1 & 16 & 5,00 & 1,40 & $-4,528$ & 37 & 0,000 \\
\hline & 2 & 23 & 5,69 & 0,00 & & & \\
\hline 26 & 1 & 16 & 3,44 & 1,55 & $-4,870$ & 37 & 0,000 \\
\hline & 2 & 23 & 5,00 & 0,00 & & & \\
\hline 27 & 1 & 16 & 2,19 & 1,05 & $-11,917$ & 37 & 0,000 \\
\hline & 2 & 23 & 4,91 & 1,29 & & & \\
\hline 28 & 1 & 16 & 2,13 & 1,09 & $-12,233$ & 37 & 0,000 \\
\hline & 2 & 23 & 4,96 & 0,21 & & & \\
\hline 29 & 1 & 16 & 2,38 & 1,41 & $-8,992$ & 37 & 0,000 \\
\hline & 2 & 23 & 5,00 & 0,00 & & & \\
\hline 30 & 1 & 16 & 2,63 & 1,45 & $-7,168$ & 37 & 0,000 \\
\hline & 2 & 23 & 4,91 & 0,42 & & & \\
\hline
\end{tabular}

\footnotetext{
a DE: desviación estándar.
}

${ }^{\mathrm{b}} \mathrm{GL}$ : grados de libertad. 
CUADRO 3. Análisis factorial exploratorio: prueba de validez conceptual del instrumento por el método de componentes principales y rotación varimax (normalización de Kaiser) y valores del coeficiente $\alpha$ de Cronbach

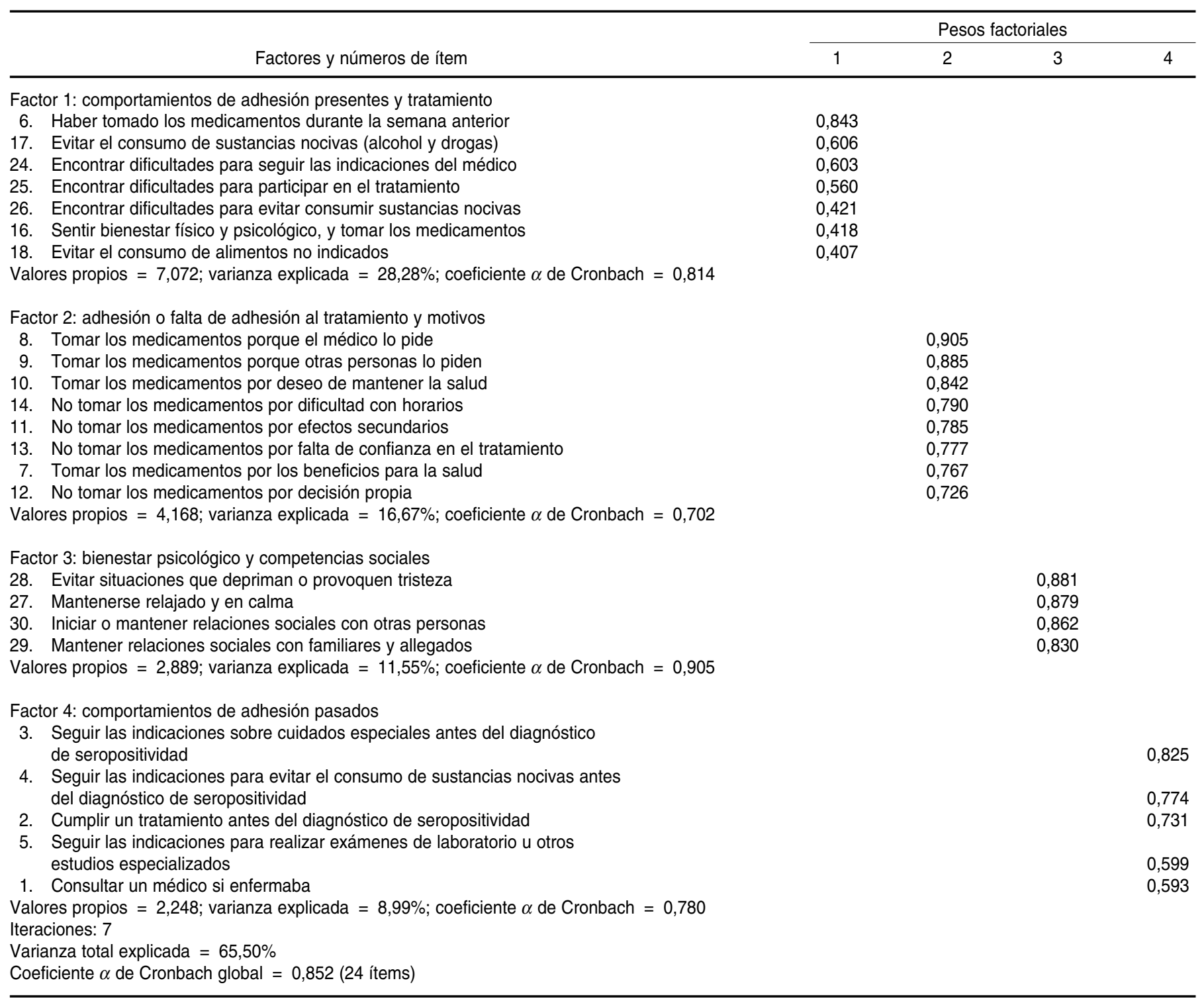

$(r=0,615 ; P<0,01)$, en el sentido de que las personas que practican comportamientos de adhesión en el presente se encuentran más motivadas para hacerlo; el factor 2 se correlacionó con el factor $3(r=-0,178 ; P<0,05)$, lo que indica que las personas menos motivadas para practicar comportamientos de adhesión presentan mejores condiciones de bienestar psicológico percibido y refieren ser más competentes en términos sociales; asimismo, el factor 2 se correlacionó con el factor $4(r=0,265 ; P<0,05)$, lo que indica que cuanto mayor es la motivación, mayor es la probabilidad de haber practicado comportamientos de adhesión en el pasado; por otro lado, se encontró una correlación entre los factores 3 y 5 ( $r=0,265 ; P<0,05)$, es decir, cuanto mayor es el tiempo de infección en meses, mayores son el bienestar psicológico percibido y el ejercicio de competencias sociales; por último, se observó una correlación entre dos variables biológicas, el recuento de linfocitos T CD4+ y la carga viral $(r=$ $-0,397 ; P<0,01)$, lo que sugiere que cuanto menor es el número de linfocitos T CD4+, mayor es la carga viral.

Por último, en el cuadro 5 se presentan los resultados del análisis de la validez predictiva. De los tres factores restantes y las variables biológicas, se encontró que los factores 2 (motivos; $\beta=0,703 ; P=0,000$ ) y 4 (comportamientos de adhesión en el pasado; $\beta=$ 0,522; $P=0,000)$ constituían factores predictivos de los comportamientos de 
CUADRO 4. Validez concurrente de variables psicológicas y comportamientos de adhesión respecto de un conjunto de variables biológicas

\begin{tabular}{lcccccc}
\hline Factores y variables biológicas & 2 & 3 & 4 & 5 & 6 & 7 \\
\hline 1. Factor 1 & $0,615^{\mathrm{a}}$ & 0,186 & 0,160 & 0,031 & 0,134 & $-0,109$ \\
2. Factor 2 & & $-0,178^{\mathrm{b}}$ & $0,265^{\mathrm{b}}$ & $-0,014$ & 0,038 & $-0,097$ \\
3. Factor 3 & & & $-0,050$ & $0,265^{\mathrm{b}}$ & 0,039 & $-0,032$ \\
4. Factor 4 & & & & 0,076 & 0,154 & 0,099 \\
5. Tiempo de infección & & & & & & \\
6. Linfocitos T CD4+ & & & & & $-0,158$ & \\
7. Carga viral & & & & & \\
\hline a $P<0,01$. & & & & & \\
b $P<0,05$. &
\end{tabular}

CUADRO 5. Validez predictiva de los factores del instrumento y las medidas biológicas respecto de los comportamientos de adhesión en el presente

\begin{tabular}{lrrr}
\hline \multicolumn{1}{c}{ Factores y variables biológicas } & \multicolumn{1}{c}{$t$} & $P$ \\
\hline Constante & & 10,607 & 0,000 \\
Factor 2 (Motivos) & 0,703 & 7,421 & 0,000 \\
Factor 4 (Comportamientos de adhesión pasados) & 0,522 & 4,286 & 0,000 \\
Factor 3 (Bienestar psicológico y competencias sociales) & $-0,137$ & $-1,450$ & 0,352 \\
Tiempo de infección & $-0,069$ & $-0,754$ & 0,454 \\
\hline
\end{tabular}

adhesión en el presente, con un coeficiente de determinación $\left(R^{2}\right)$ ajustado de 0,486 (estos dos factores explican, en conjunto, un $48,6 \%$ de la varianza).

\section{DISCUSIÓN}

Tal y como se mencionó antes, una de las principales preocupaciones de los profesionales de la salud que atienden pacientes infectados por el VIH es la imperiosa necesidad de contar con recursos metodológicos confiables y válidos que permitan medir la frecuencia con la que se practican los comportamientos de adhesión al tratamiento y las variables que facilitan o impiden que dichos comportamientos se practiquen según las instrucciones del equipo sanitario $(7,8)$. En este contexto, los resultados obtenidos al aplicar el VPAD-24 a un grupo de personas seropositivas indican que este instrumento posee óptimas propiedades psicométricas de confiabilidad y validez.

En la literatura especializada se describe el empleo de diversos instrumentos para medir la adhesión al tratamiento de pacientes seropositivos (8, 19,35-36), pero el instrumento presentado en este estudio se diferencia en tres aspectos de los utilizados por otros autores.

En primer lugar, el VPAD-24 se basa en un modelo psicológico de salud biológica $\mathrm{y}$, en consecuencia, permite delimitar con precisión la dimensión psicológica que es pertinente a la salud en general y al problema de la adhesión al tratamiento en particular.

En segundo lugar, para su construcción se consideró la eventual relación entre los factores que incluye y otras variables de relevancia teórica, como por ejemplo, las situaciones vinculadas con el estrés y los comportamien- tos asociados a la enfermedad (véase la figura 1). Deseamos así subrayar que la construcción de instrumentos debe pasar, desde nuestra óptica, por el análisis y la discusión teórica y conceptual de las variables que pueden ser pertinentes para abordar el problema en cuestión, pues solo cumplido este requisito se estará en una posición inmejorable para explicar o predecir, según sea el caso, por qué, en términos psicológicos, las personas practican o no comportamientos de adhesión.

En tercer y último lugar, este instrumento no solo mide comportamientos de adhesión circunscritos exclusivamente al consumo de medicamentos, lo que, en nuestra opinión, le proporciona un valor agregado. En efecto, estos comportamientos no son los únicos que hay que tener en cuenta cuando se diseña un plan de intervención, en el cual también hay que considerar otros comportamientos relacio- 
FIGURA 1. Representación conceptual del modelo psicológico aplicado a la adhesión al tratamiento de personas seropositivas frente al VIH

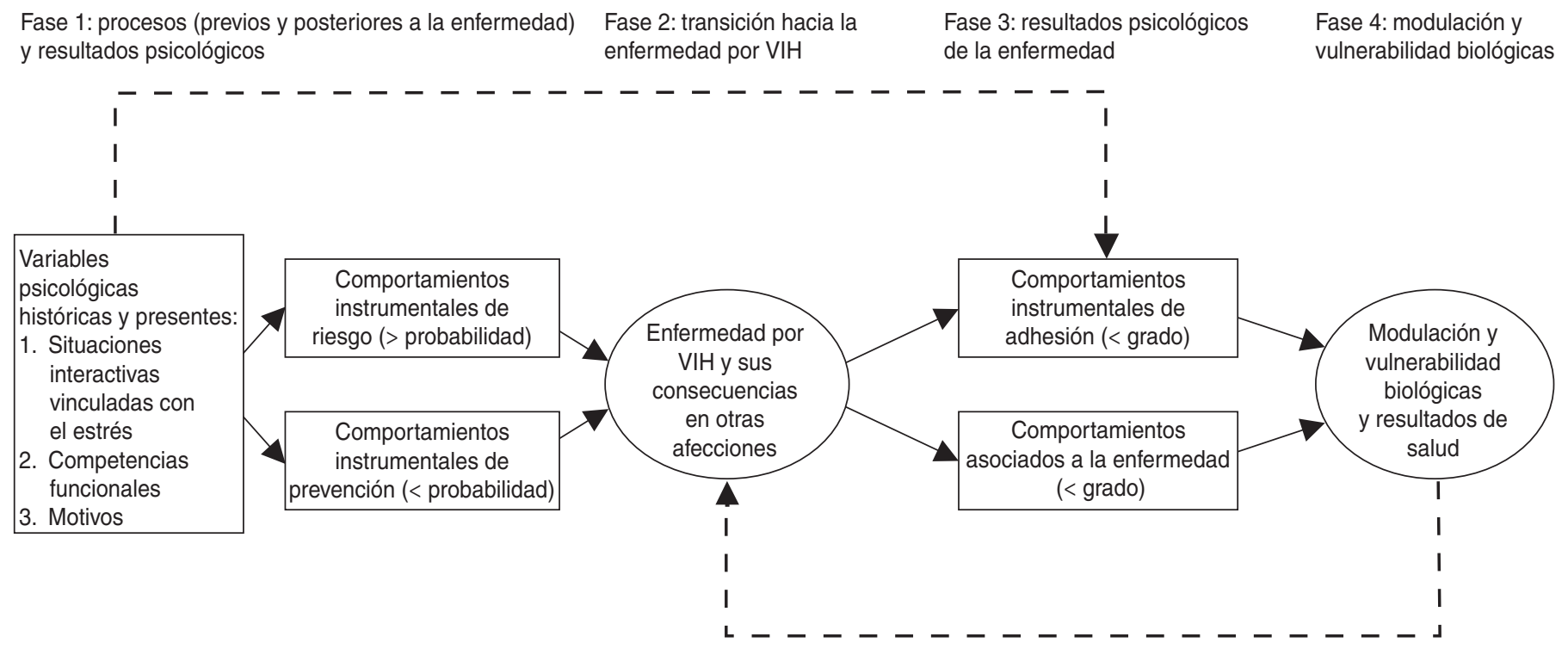

nados con restricciones en la dieta y abstención de consumir sustancias como alcohol o drogas (25).

De la misma manera se pueden comentar las variables analizadas, particularmente dos de ellas: los comportamientos de adhesión en el pasado (previos al diagnóstico de seropositividad al VIH) y los motivos que subyacen tras la práctica o la omisión de comportamientos de adhesión. En efecto, si partimos del supuesto de que estos últimos no ocurren de manera aislada, sino que pueden ser la manifestación de una diversidad de variables psicológicas históricas (competencias pasadas) y situacionales (motivos, estrés y comportamientos asociados a la enfermedad, entre otras) $(6,7,30,37-41)$ tal como se demostró en el análisis de regresión, ambas variables constituyen factores predictivos de los comportamientos de adhesión en el presente.

Dicho en otras palabras, en la medida en que se dispone de los recursos competenciales, o sea, se tienen conocimientos acerca de algo (información sobre el sida, los medicamentos, los posibles efectos secundarios, etcétera) y se hace algo porque ya se ha hecho en el pasado, es más probable que esos recursos se traduzcan en comportamientos de adhesión en el presente, como tomar los medicamentos, consumir los alimentos indicados y evitar el consumo de sustancias nocivas. Si además los pacientes seropositivos están motivados para participar activamente en el plan de intervención y seguir el conjunto de instrucciones expuestas por el personal sanitario, cabe esperar, en cuanto a su estado de salud, que se incremente el número de linfocitos CD4+ y se mantenga baja la carga viral, dos de los indicadores biológicos por excelencia en esta infección.

A pesar del aspecto prometedor de los resultados presentados, es importante hacer notar algunas limitaciones. La primera de ellas se relaciona con el tamaño de la muestra. Aunque se trabajó con una muestra bastante representativa - poco más del $75 \%$ del total de personas seropositivas que en el momento del estudio eran atendidas en el Centro de Información para la Salud-, es necesario repetir el estudio con grupos de pacientes seropositivos atendidos en otras dos instituciones sanitarias del estado de Sonora, para confirmar los resultados referidos en este artículo.
En segundo lugar, llama nuestra atención el grupo de variables excluidas de los análisis, sobre todo las relacionadas con la evaluación que las personas seropositivas hacen de la información sobre la enfermedad, el tratamiento, los estudios de laboratorio, etcétera, las cuales presumiblemente podrían ser indicadoras de cierto tipo de conocimiento, posteriormente pertinente para la adopción y la práctica de comportamientos de adhesión. Esto hace pensar que tal vez sean variables que podrían haber derivado en un mayor poder de predicción de esos comportamientos.

En tercer lugar, destaca la ausencia de una influencia, en general, de las variables biológicas, tanto en los análisis realizados para probar la validez concurrente como en los relativos a la validez predictiva. Así, aunque el presente estudio se llevó a cabo con un propósito particular, es preciso analizar en trabajos futuros la relación entre las variables psicológicas aquí consideradas $\mathrm{u}$ otras más (situaciones vinculadas con estrés y comportamientos asociados a la enfermedad, entre otras) y las variables biológicas y el control o la progresión clínica de la enfermedad. 


\section{REFERENCIAS}

1. Lavalle C, David JC, Peña F, Estrada-Aguilar JL, Aviña-Zubieta JA, Madrazo M. Reduction in hospitalization cost, morbidity, disability, and mortality in patients with AIDS treated with protease inhibitors. Arch Med Res. 2000; 31:515-9.

2. Murphy EL, Collier AC, Kalish LA, Assmann SF, Para MF, Flanigan TP, et al. Highly active antiretroviral therapy decreased mortality and morbidity in patients with advanced HIV disease. Ann Intern Med. 2001;135:17-26.

3. Palella FJ, Delaney KM, Moorman AC, Loveless MO, Fuhrer J, Satten GA, et al. Declining morbidity and mortality among patients with advanced human immunodeficiency virus infection. New Engl J Med. 1998;338:853-60.

4. Grabar S, Moing V, Goujard C, Egger M, Leport C, Kazatchkine M, et al. Response to highly active antiretroviral therapy at 6 months and long-term disease progression in HIV-1 infection. J Acquir Immune Defic Syndr. 2005;39:284-92.

5. Rangel-Frausto S. Tratamiento antirretroviral. En: Ponce de León S, Rangel-Frausto S. SIDA. Aspectos clínicos y terapéuticos. México, D.F.: McGraw-Hill Interamericana; 2000. Pp. 441-54.

6. Bayés R. Aspectos psicosociales en la adhesión al tratamiento antirretrovírico en la infección por VIH. Publicación Oficial de la Sociedad Española Interdisciplinaria del SIDA. 1999;10:165-6.

7. Bayés R. Infección por VIH: aspectos psicológicos. En: Bueno F, Nájera R. Salud Pública y SIDA. Madrid: Ediciones Doyma; 2001: 479-86.

8. Remor EA Valoración de la adhesión al tratamiento antirretroviral en pacientes VIH+. Psicothema. 2002;14:262-7.

9. Sánchez-Sosa JJ. Treatment adherence: The role of behavioral mechanisms and some implications for health care interventions. Rev Mex Psic. 2002;19:85-92.

10. Bogart LM, Catz SL, Kelly JA, Gray-Bernhardt ML, Hartmann BR, Otto-Salaj LL, et al. Psychosocial issues in the era of new AIDS treatments from the perspective of persons living with HIV. J Health Psychol. 2000;5:500-16.

11. Kalichman SC, Catz S, Ramachandran B. Barriers to HIV/AIDS treatment and treatment adherence among African-American adults with disadvantaged education. JAMA. 1999; 91:439-46.

12. Meystre-Agustoni G, Dubois-Archer F, Cochand $\mathrm{P}$, Telenti A. Antiretroviral therapies from the patients perspective. AIDS Care. 2000;12:717-21.

13. Siegel K, Schrimshaw EW. Stress, appraisal, and coping: a comparison of HIV-infected women in the pre-HAART and HAART eras. J Psychosom Res. 2005;58:225-33.

14. Murphy DA, Marelich WD, Hoffman D Steers WN. Predictors of antiretroviral therapy. AIDS Care. 2005;16:471-84.

15. Safren SA, Radomsky AS, Otto MW, Salomon E. Predictors of psychological well-being in a diverse sample of HIV-positive patients receiving highly active antiretroviral therapy. Psychosomatics. 2002;43:478-85.

16. Summers J, Robinson R, Capps L, Zisook S, Hampton J, McCutchan E, et al. The influence of HIV-related support groups on survival in women who live with HIV. Psychosomatics. 2000;41: 262-8.

17. Hinkin $\mathrm{CH}$, Castellon SA, Durvasula RS, Hardy DJ, Lam MN, Mason KI, et al. Medication adherence among HIV+ adults: effects of cognitive dysfunction and regimen complexity. Neurol. 2002;59:1944-50.

18. Codina C, Tuset M, Martínez M, del Cacho E, Miró JM, Ribas J. Cómo mejorar la adhesión al tratamiento antirretroviral. Medicina Integral. 2001;37:482-6.

19. Wagner GJ, Remien RH, Carballo-Diéguez A Correlates of adherence to combination antiretroviral therapy among members of HIVpositive mixed status couples. AIDS Care 2002. 14:105.

20. Hosek SG, Harper GW, Domanico R. Predictors of medication adherence among HIVinfected youth. Psychol Health Med. 2005;10: $166-79$.

21. Molassiotis A. A pilot study of the effects of a behavioural intervention on treatment adherence in HIV-infected patients. AIDS Care. 2003;15:125-35.

22. Reynolds NR. The problem of antiretroviral adherence: a self-regulatory model for intervention. AIDS Care. 2003;15:117-24.

23. Tobón S, Vinaccia S. Modelo de intervención psicológica en el VIH/sida. Psicol Salud. 2003;13:161-74.

24. World Health Organization. Adherence to log-term therapies. Evidence for action. Geneva; 2003. (WHO/MNC/03.01).

25. DiMatteo MR. Variations in patients' adherence to medical recommendations. A quantitative review of 50 years of research. Med Care. 2004;42:200-9.

26. Ostenberg L, Blaschke T. Adherence to medication. New Engl J Med. 2005;353:487-97.

27. Liu H, Golin CE, Miller LG, Hays RD, Beck K, Sanadaji $S$, et al. A comparison study of multiple measures of adherence to HIV protease inhibitors. Ann Intern Med. 2001; 134:968-77.
28. Piña JA. Intervención psicológica en el área de la infección por el VIH: obstáculos y retos para los psicólogos de la salud. Terapia Psicol. 2003;21:23-7.

29. Piña JA. Psicología y VIH/sida: aportaciones y asignaturas pendientes en México. Higiene. 2003;5:184-9.

30. Ribes E. Psicología y salud: un análisis conceptual. Barcelona: Martínez Roca; 1990.

31. Bayés R, Ribes E. Un modelo psicológico de prevención de enfermedad y su aplicación al caso del SIDA. En: Piña JA. Psicología y salud: aportes del análisis de la conducta. Hermosillo, México: Editorial UNISON; 1992. Pp. 1-21.

32. Silva A. La investigación asistida por computadora. México, D.F.: Universidad Nacional Autónoma de México; 1998.

33. Corral V, Frías M, González D. Análisis cuantitativo de variables manifiestas. Textos académicos N. ${ }^{\circ} 11$. Hermosillo, México: Editorial UNISON; 2001

34. Corral V, Frías M, González D. Análisis cuantitativo de variables manifiestas. Textos académicos N. ${ }^{\circ} 13$. Hermosillo, México: Editorial UNISON; 2001.

35. Catz SL, Heckman TG, Kochman A, DiMarco M. Rates and correlates of HIV treatment adherence among late middle-aged and older adults living with HIV disease. Psychol Health Med. 2001;6:47-58.

36. Knobel H, Alonso J, Casado JL, Collazos J, González J, Ruiz I, et al. Validation of a simplified medication adherence questionnaire in a large cohort of HIV-infected patients: the GEEMA study. AIDS. 2002;16:605-13.

37. Gallant JE. Strategies for long-term success in the treatment of HIV infection. JAMA. 2000; 1329-34.

38. Stone VE, Hogan JW, Schuman P, Rompalo AM, Howard A, Korkontzelou C, et al. Antiretroviral regimen complexity, self-reported adherence, and HIV patients' understanding of their regimens: Survey of women in the HER study. J Acquir Immune Defic Syndr. 2001;28:124-31.

39. Aloisi MS, Arici C, Balzano R, Noto P, Piscopo $R$, Filice $G$, et al. Behavioral correlates of adherence to antiretroviral therapy. J Acquir Immune Defic Syndr. 2002;31(suppl. 3): S145-S8.

40. Bottonari KA, Roberts JE, Ciesla JA, Hewitt RG. Life stress and adherence to antiretroviral therapy among HIV-positive individuals: a preliminary investigation. AIDS Patient Care STDS. 2005;19:719-27.

41. Siegel K, Lehas HM. AIDS as chronic illness: psychosocial implications. AIDS. 2002;16 (suppl. 4):S69-S76. 


\section{ANEXO 1. Encuesta para medir variables psicológicas y comportamientos de adhesión al tratamiento en personas $\mathrm{VIH}+(\mathrm{VPAD}-30)$}

Las preguntas de la 1 a la 5 tienen que ver con si usted practicaba y con qué frecuencia diferentes comportamientos antes de que a usted le notificaran su condición como persona $\mathrm{VIH+}$

1. Antes de recibir el diagnóstico como persona $\mathrm{VIH}+$, si usted se sentía mal físicamente o enfermaba, por ejemplo, del estómago, de gripe, de la garganta, de bronquitis, etcétera, ¿acudía con un médico para que la revisara y le diera tratamiento?

(5) Siempre

(4) La mayoría de las veces

(3) En general, la mitad de las veces

_ (2) Muy pocas veces

(1) Nunca

2. Antes de recibir el diagnóstico como persona $\mathrm{VIH}+$, si usted acudía con su médico y éste le daba algún tratamiento, ¿seguía usted las instrucciones que le daban al pie de la letra, es decir, consumía los medicamentos tal y como se lo indicó su médico?

(5) Siempre

- (4) La mayoría de las veces

(3) En general, la mitad de las veces

(2) Muy pocas veces

(1) Nunca

3. Antes de recibir el diagnóstico como persona $\mathrm{VIH}+$, si usted enfermaba y al acudir con su médico éste le pedía, por ejemplo, que guardara reposo o que descansara en casa, ¿seguía usted las instrucciones que le daba su médico?

(5) Siempre

(4) La mayoría de las veces

(3) En general, la mitad de las veces

(2) Muy pocas veces

(1) Nunca

4. Antes de recibir el diagnóstico como persona $\mathrm{VIH}+$, si usted enfermaba y su médico le pedía que evitara el consumo de determinados alimentos o de sustancias como alcohol, por ejemplo, ¿seguía usted las indicaciones que le daba su médico?

(5) Siempre

(4) La mayoría de las veces

(3) En general, la mitad de las veces

_ (2) Muy pocas veces

(1) Nunca

5. Antes de recibir el diagnóstico como persona $\mathrm{VIH}+$, si su médico le pedía que se realizara estudios diversos de laboratorio (sangre, orina, excremento, rayos $\mathrm{x}$, etcétera), ¿seguía usted las indicaciones que le daba su médico?

(5) Siempre

- (4) La mayoría de las veces

(3) En general, la mitad de las veces

(2) Muy pocas veces

- (1) Nunca

Las preguntas de la 6 a la 18 tienen que ver con variables psicológicas que se relacionan con la práctica de diferentes comportamientos, a partir de que a usted se le diagnosticó como persona $\mathrm{VIH}+$

6. En el transcurso de la semana pasada, ¿consumió usted todos los medicamentos de acuerdo con las instrucciones que le dio su médico?

(5) Todos los días de la semana (seguir con las preguntas 7, 8, 9 y 10, luego con la 15)

- (4) La mayoría de los días de la semana (seguir con las preguntas 11, 12, 13 y 14, luego con la 15)

(3) En general, la mitad de los días de la semana (seguir con las preguntas 11, 12, 13 y 14, luego con la 15)

- (2) Muy pocos días de la semana (seguir con las preguntas 11, 12, 13 y 14, luego con la 15)

_ (1) Ningún día de la semana (seguir con las preguntas 11, 12, 13 y 14, luego con la 15)

Si usted siguió las instrucciones de su médico y consumió los medicamentos todos los días de la semana, ¿nos podría decir qué tan determinante fue cada uno de los motivos que se mencionan?

7. He experimentado sus beneficios

(4) Fue un motivo bastante determinante

(3) Fue un motivo más o menos determinante

(2) Fue un motivo poco determinante

(1) No fue un motivo determinante

8. Porque el médico me lo pidió

(4) Fue un motivo bastante determinante

- (3) Fue un motivo más o menos determinante

(2) Fue un motivo poco determinante

(1) No fue un motivo determinante

9. Lo hice por personas cercanas a $\mathrm{mi}$

(4) Fue un motivo bastante determinante

_ (3) Fue un motivo más o menos determinante

(2) Fue un motivo poco determinante

_ (1) No fue un motivo determinante 
10. Porque tengo deseos de mantenerme saludable el mayor tiempo posible

(4) Fue un motivo bastante determinante

- (3) Fue un motivo más o menos determinante

_ (2) Fue un motivo poco determinante

- (1) No fue un motivo determinante

Si usted no siguió las instrucciones de su médico, es decir, que NO consumió los medicamentos todos los días de la semana, ¿nos podría decir cuál fue el motivo y qué tan determinante fue cada uno de ellos?

11. Me provocan efectos secundarios demasiado molestos

(4) Fue un motivo bastante determinante

_ (3) Fue un motivo más o menos determinante

(2) Fue un motivo poco determinante

(1) No fue un motivo determinante

12. Por voluntad o decisión propia

(4) Fue un motivo bastante determinante

- (3) Fue un motivo más o menos determinante

_ (2) Fue un motivo poco determinante

(1) No fue un motivo determinante

13. No tengo confianza en los beneficios de los medicamentos

(4) Fue un motivo bastante determinante

- (3) Fue un motivo más o menos determinante

- (2) Fue un motivo poco determinante

(1) No fue un motivo determinante

14. Por dificultades con los horarios

(4) Fue un motivo bastante determinante

- (3) Fue un motivo más o menos determinante

_ (2) Fue un motivo poco determinante

(1) No fue un motivo determinante

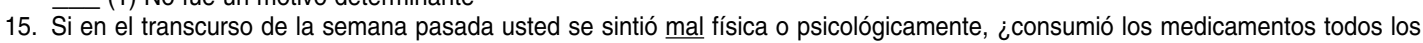
días de acuerdo con las instrucciones de su médico?

_ (5) Todos los días de la semana

(4) La mayoría de los días de la semana

_ (3) En general, la mitad de los días de la semana

- (2) Muy pocos días de la semana

(1) Ningún día de la semana

16. Si en el transcurso de la semana usted se sintió bien física o psicológicamente, ¿consumió los medicamentos todos los días de acuerdo con las instrucciones de su médico?

(5) Todos los días de la semana

- (4) La mayoría de los días de la semana

(3) En general, la mitad de los días de la semana

- (2) Muy pocos días de la semana

(1) Ningún día de la semana

17. En el transcurso de la semana pasada, ¿consumió usted sustancias como alcohol o drogas, a pesar de saber que pueden afectar el tratamiento?

(5) Ningún día de la semana

- (4) Muy pocos días de la semana

(3) En general, la mitad de los días de la semana

(2) La mayoría de los días de la semana

(1) Todos los días de la semana

18. En el transcurso de la semana pasada, ¿consumió usted algún tipo de alimento que le recomendaron no consumiera por razones especiales relacionadas con el tratamiento?

(5) Ningún día de la semana

(4) Muy pocos días de la semana

(3) En general, la mitad de los días de la semana

(2) La mayoría de los días de la semana

— (1) Todos los días de la semana

Las preguntas de la 19 a la 22 tienen que ver con la forma en la que usted evalúa diferentes asuntos relacionados con la enfermedad y el tratamiento

19. ¿Cómo evalúa la información que tiene sobre la enfermedad?

(5) Bastante completa

(4) Completa, a secas

(3) Regular

(2) Muy escasa

(1) No tengo información

20. ¿Cómo evalúa la información que tiene sobre el tratamiento y los medicamentos que consume actualmente?

(5) Bastante completa

(4) Completa, a secas 
(3) Regular

_ (2) Muy escasa

(1) No tengo información

21. ¿Cómo evalúa la información que tiene sobre los resultados de los estudios de laboratorio y, sobre todo, lo que significan?

(5) Bastante completa

(4) Completa, a secas

- (3) Regular

(2) Muy escasa

(1) No tengo información

22. ¿Cómo evalúa la información que le proporciona su médico sobre la enfermedad y el tratamiento?

(5) Bastante completa

— (4) Completa, a secas

(3) Regular

- (2) Muy escasa

_ (1) No tengo información

Las preguntas de la $\mathbf{2 3}$ a la $\mathbf{3 0}$ tienen que ver con la forma en la que usted evalúa el grado de dificultad para practicar diferentes comportamientos

23. ¿Qué tan difícil se le hace buscar información relacionada con su enfermedad y el tratamiento, por ejemplo?

(5) Nada difícil

- (4) Un poco difícil

(3) Más o menos difícil

- (2) Difícil, secas

(1) Bastante difícil

24. ¿Qué tan difícil se le hace a usted seguir con las instrucciones que le proporciona su médico en relación con el consumo de medicamentos?

(5) Nada difícil

- (4) Un poco difícil

- (3) Más o menos difícil

- (2) Difícil, secas

(1) Bastante difícil

25. ¿Qué tan difícil se le hace a usted participar de manera activa, junto con su médico, en la toma de decisiones sobre su plan de tratamiento?

(5) Nada difícil

(4) Un poco difícil

— (3) Más o menos difícil

(2) Difícil, secas

(1) Bastante difícil

26. ¿Qué tan difícil se le hace a usted dejar de consumir sustancias como alcohol o drogas?

(5) Nada difícil

— (4) Un poco difícil

(3) Más o menos difícil

— (2) Difícil, secas

(1) Bastante difícil

27. ¿Qué tan difícil se le hace a usted mantenerse relajado y en calma?

(5) Nada difícil

(4) Un poco difícil

— (3) Más o menos difícil

(2) Difícil, secas

(1) Bastante difícil

28. ¿Qué tan difícil se le hace a usted evitar situaciones que le provoquen tristeza o que lo depriman?

(5) Nada difícil

_ (4) Un poco difícil

— (3) Más o menos difícil

_ (2) Difícil, secas

(1) Bastante difícil

29. ¿Qué tan difícil se le hace a usted mantener sus relaciones sociales con personas allegadas, como sus familiares, por ejemplo?

(5) Nada difícil

(4) Un poco difícil

(3) Más o menos difícil

- (2) Difícil, secas

(1) Bastante difícil

30. ¿Qué tan difícil se le hace a usted mantener o iniciar nuevas relaciones de amistad?

(5) Nada difícil

- (4) Un poco difícil

— (3) Más o menos difícil

(2) Difícil, secas

— (1) Bastante difícil 
ABSTRACT Objective. To test the psychometric properties of an instrument for measuring psychological variables and adherence to treatment behaviors in persons with HIV infection or AIDS.

Instrument for measuring psychological variables and adherence to treatment behavior in persons who are seropositive for HIV (VPAD-24)
Methods. A cross-sectional study was performed with 68 subjects who were seropositive for the human immunodeficiency virus (HIV) and who were being treated in a health facility in the city of Hermosillo (Sonora), Mexico, at the time the study was carried out. Five consecutive statistical testing procedures were applied for data analysis: the Student $t$ test for comparison of means, for assessing the comprehensibility of the questions; an exploratory factorial analysis with varimax rotation for determining construct validity; Cronbach's $\alpha$ coefficient for determining reliability; Pearson's correlation coefficient $(r$ ) for assessing concurrent validity; and multiple linear regression for determining predictive value.

Results. In 25 out of a total of 30 questions, the Student $t$ test for comparison of means gave statistical significance values of $P \leq 0.05, P \leq 0.005, P \leq 0.01$, and $P \leq 0.001$. A four-factor structure was found with the factorial analysis with varimax rotation: (1) current adherence behavior and treatment; (2) underlying reasons for adherence or lack of adherence; (3) psychological well-being and social competencies; (4) past adherence behavior with values above 1 that, taken as a whole, explain $65.50 \%$ of the variance. An overall $\alpha$ coefficient of 0.852 was obtained with the test for reliability. The test for concurrent validity showed correlations between factors 1 and $2(r=0.615$; $P=<0.01)$, between factor 2 and factors 3 and $4(r=-0.178 ; P<0.05$ and $r=0.265$; $P<0,05$, respectively), and between factor 3 and duration of infection in months ( $r=$ $0.265 ; P<0.05)$. Finally, multiple linear regression showed that psychological factors 2 and 4 , which together explain $48.6 \%$ of the variance, predicted current adherence behavior (adjusted determination coefficient $\left[R^{2}\right]=0.486$ ).

Conclusion. According to the results obtained, the instrument has optimal reliability as well as construct validity, concurrent validity, and predictive validity.

Key words Patient cooperation, seropositivity, reproducibility of results, validity, Mexico.

\section{Convocatoria a la propuesta de estudios para el Premio Fred L. Soper 2006}

La Fundación Panamericana de la Salud y la Educación (PAHEF) solicita propuestas para el Premio Fred L. Soper, otorgado anualmente a un artículo de investigación que aporte conocimientos novedosos sobre temas de salud pública de interés para América Latina 0 el Caribe. Los estudios propuestos para el premio que se otorgará en 2006 deberán haberse publicado en 2005 en revistas científicas incluidas en el Index Medicus o en las publicaciones oficiales de la Organización Panamericana de la Salud (OPS), y sus autores deberán estar afiliados a instituciones docentes, de investigación o de servicios ubicadas en países de América Latina 0 el Caribe, incluidos los centros colaboradores de la OPS. Los estudios pueden ser experimentales o de observación y consistir en análisis de datos nuevos o en revisiones de datos primarios, pero interesan sobre todo los de naturaleza multidisciplinaria y los que versan sobre enfermedades infecciosas, que eran el campo de interés particular del Dr. Fred L. Soper, Director de la OPS de 1947 a 1958 y uno de los salubristas más destacados del siglo XX. Cualquiera puede proponer un trabajo como candidato al premio, incluso sus propios autores.

PAHEF maneja el fondo dedicado al premio, que consiste en un certificado y US $\$ 2.500$ en efectivo, y mantiene una cuenta especial para donaciones voluntarias. Los finalistas son elegidos por el Comité del Premio, cuyos miembros son designados por la OPS y PAHEF. Todas las candidaturas deberán recibirse a más tardar el 25 de agosto de 2006.

Dirección para el envío de las propuestas:

Comité de Selección Premio Fred L. Soper

Fundación Panamericana de la Salud y Educación

525 Twenty-third Street, N.W.

Washington, D.C. 20037, U.S.A.

Tel.: 202-974-3416

Fax: 202-974-3636

Correo electrónico: pahef@paho.org

Internet: http://www.paho.org/Spanish/PAHEF/soper.htm 